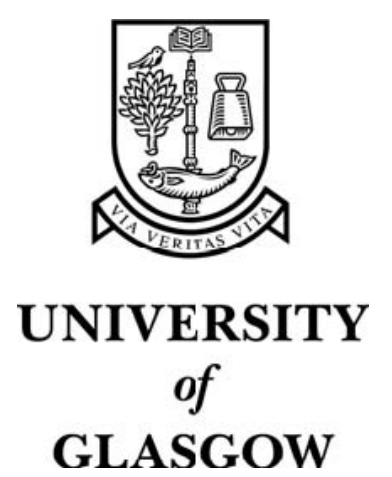

Schauer, T. and Negård, N. -O. and Previdi, F. and Hunt, K. J. and Fraser, M. H. and Ferchland, E. and Raisch, J. (2005) Online identification and nonlinear control of the electrically stimulated quadriceps muscle. Control Engineering Practice 13(9):pp. 1207-1219.

http://eprints.gla.ac.uk/3363/ 


\title{
Online Identification and Nonlinear Control of the Electrically Stimulated Quadriceps Muscle
}

\author{
T. Schauer a,d,*, N.-O. Negård ${ }^{\mathrm{a}, \mathrm{b}}$, F. Previdi ${ }^{\mathrm{c}}$, K.J. Hunt ${ }^{\mathrm{d}, \mathrm{e}}$, \\ M.H. Fraser ${ }^{\mathrm{e}}$, E. Ferchland ${ }^{\mathrm{a}}$, J. Raisch ${ }^{\mathrm{b}, \mathrm{a}}$ \\ a Max Planck Institute for Dynamics of Complex Technical Systems, Sandtorstr. 1, \\ 39106 Magdeburg, Germany \\ ${ }^{\mathrm{b}}$ Lehrstuhl für Systemtheorie technischer Prozesse, Institut für \\ Automatisierungstechnik, Universität Magdeburg, Postfach 4120, 39016 \\ Magdeburg, Germany \\ ${ }^{\mathrm{c}}$ Dipartimento di Ingegneria, Universitá di Bergamo, via Marconi 5, 24044 \\ Dalmine (BG), Italy \\ ${ }^{\mathrm{d}}$ Centre for Rehabilitation Engineering, University of Glasgow, \\ Glasgow G12 8QQ, Scotland, UK

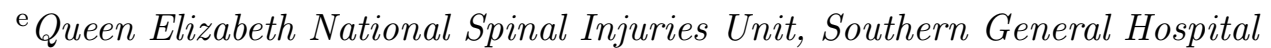 \\ 1345 Govan Road, Glasgow G51 4FT, Scotland, UK
}

\begin{abstract}
A new approach for estimating nonlinear models of the electrically stimulated quadriceps muscle group under non-isometric conditions is investigated. The model can be used for designing controlled neuro-prostheses. In order to identify the muscle dynamics (stimulation pulsewidth - active knee moment relation) from discrete-time angle measurements only, a hybrid model structure is postulated for the shankquadriceps dynamics. The model consists of a relatively well known time-invariant passive component and an uncertain time-variant active component. Rigid body dynamics, described by the Equation of Motion (EoM), and passive joint properties form the time-invariant part. The actuator, i.e. the electrically stimulated muscle group, represents the uncertain time-varying section. A recursive algorithm is outlined for identifying online the stimulated quadriceps muscle group. The algorithm requires EoM and passive joint characteristics to be known a priori. The muscle dynamics represent the product of a continuous-time nonlinear activation dynamics and a nonlinear static contraction function described by a Normalised Radial Basis Function (NRBF) network which has knee-joint angle and angular velocity as input arguments. An Extended Kalman Filter (EKF) approach is chosen to estimate muscle dynamics parameters and to obtain full state estimates of the shank-quadriceps dynamics simultaneously. The latter is important for implementing state feedback controllers. A nonlinear state feedback controller using the backstepping method is
\end{abstract}


explicitly designed whereas the model was identified a priori using the developed identification procedure.

Key words: Electrical stimulation, Extended Kalman filter, Physiological model, Neural network, Nonlinear control

\section{Introduction}

The development of controlled neuro-prostheses for the restoration of basic motor functions to paraplegics represents a major research area in rehabilitation engineering. These lower limb prostheses are based on a technique called Functional Electrical Stimulation (FES), in which stimulation of paralysed muscles can generate muscle contractions (Popović and Sinkjær, 2000). FES, together with appropriate sensor and control technology, can provide useful, controlled functional movement. To design controllers for FES systems, it can be useful to have an accurate model which describes the relation between the stimulation parameters and the resulting force, moment or movement.

In this paper we are particularly interested in a physiologically-based nonlinear modelling approach for the electrically stimulated quadriceps muscle group under non-isometric conditions (changing muscle length). The experimental set-up will be given in Section 2. As an alternative, black-box modelling approaches can be considered (Gollee and Hunt, 1997; Previdi, 2002; Schauer et al., 2001; Schauer and Hunt, 2000). However, in nonlinear black-box model design, depending on the model family used, structure selection could be a difficult task. In particular, to obtain an effective structure identification long measurements time series could be needed.

When modelling this dynamics on a physiological basis, the combination of a modified Hill-type muscle model and a passive model part is often assumed. The latter consists of the Equation of Motion (EoM), passive elastic and passive viscous properties; first principles, simple pendulum tests and static force measurements without stimulation are used to obtain this model part.

Detailed muscle models describe submuscle groups of the quadriceps muscle group separately (e.g. (Riener and Fuhr, 1998)). Assuming a standard Hill-type muscle model (Hill, 1938, 1970), the generated muscle force of the individual muscle groups is the product of muscle activation (described by a

* Corresponding author. Phone +49 3916110 431, Fax +49 3916110601

Email address: schauer@mpi-magdeburg.mpg.de (T. Schauer ). 
nonlinear recruitment curve and a linear dynamics with time delay), a nonlinear force - muscle length relation and a nonlinear force - muscle contraction velocity relation. To calculate the resulting knee moment, nonlinear moment arms (parameterised by the joint angle) for the muscles with respect to the knee-joint have to be known. Passive muscle properties are usually assigned to the joint. The identification of such muscle models is not possible or too complicated in vivo, so that parameters are often taken from literature that leads to a bad fit of the models to individual subjects.

Alternatively, the quadriceps can be treated as a single muscle, and joint moment generation can be directly described in the joint angle - angular velocity space instead of the muscle length - muscle contraction velocity space. To keep the identification task simple, often linear moment - joint angle and moment - angular velocity relations are postulated, which are only valid in a limited subrange of the entire joint angle - velocity range.

Identification of the quadriceps dynamics (stimulation - active knee moment relation) from shank movements was investigated in Chizeck et al. (1999) (online estimation) and in Franken et al. (1995); Ferrarin and Pedotti (2000) (off-line estimation). All these approaches require beside angle information either direct measurements of velocity and acceleration or their numerical computation from frequently sampled knee-joint angle measurements.

In Section 3, we present a simple approach based on an Extended Kalman Filter to estimate a continuous-time nonlinear muscle model and its internal states from discrete-time knee-joint angle measurements only, sampled with stimulation frequency. Further, we look for a general nonlinear function that describes the active knee moment as a function of joint angle and angular velocity. The identification method will be demonstrated with simulations and analysis of real data.

Further we show in Section 4 the usefulness of the proposed identification procedure to derive nonlinear models for controller design. As the EKF delivers state estimates directly, the application of state feedback for controlling the knee-joint position is appealing.

The knee-joint tracking is simple to perform in experiments, and many researchers have addressed this problem. It is also a popular benchmark, because it can give us answers about the limitations and possibilities of closed-loop control applied to physiological systems.

However, such a knee-joint controller may also be used in real situations: Before carrying out functional tasks like standing-up or cycling using FES, paraplegics usually undergo an exercise regime to strengthen and rebuild their paralysed muscles. This is often performed under non-isometric conditions while generating cyclic movements such as lifting the shank. Since optimal 
stimulation patterns are difficult to determine and dependent on subject and day, fixed patterns such as ramps are applied in clinical use and implemented open-loop in available stimulation hardware. Such patterns have the drawback that changing muscle conditions due to fatigue and disturbances cannot be taken into account.

By sensing relevant information on the musculoskeletal state the stimulation pattern can be adapted online. The desired movement can have an arbitrary shape over time. In a training session the angle profile can be easily re-programmed. We therefore do not need to rely on open-loop adjustment of the stimulation pattern and this helps to avoid dangerous situations such as hyper-extension of the knee.

In recent years several articles about the control of cyclic movements have been published (e.g. PD/PID (Chang et al., 1997; Ferrarin et al., 2001; Riess and Abbas, 2000), linear polynomial controller (Schauer and Hunt, 2000), neurocontrol (Chang et al., 1997; Riess and Abbas, 2000), adaptive control (Ferrarin et al., 2001; Riess and Abbas, 2000), nonlinear feedforward control with linear feedback stabilisation using PD/PID control (Chang et al., 1997; Ferrarin et al., 2001; Riess and Abbas, 2000), directly data-driven controller design (Previdi et al., 2004), sliding mode control (Jezernik et al., 2004; Schauer et al., 2002), gain scheduling controller (Previdi and Carpanzano, 2003)). The existing linear approaches using PD/PID control have been shown to be unsuitable. These controllers were either empirically tuned or determined by standard tuning rules. Better results can be obtained by using higher order linear controllers based on identified pulse transfer functions of the system (Schauer and Hunt, 2000). Neuro-control and nonlinear control approaches can in contrast to linear control provide better performance but often require more sensory information. This problem will be mitigated by using state estimates provided by an EKF as proposed in this article. Adaptive control represents an interesting approach for time varying systems like the human body. The essential stability proof for the closed-loop is however highly problematical for nonlinear adaptive systems. These might also be a problem when using EKFs as convergence of the parameter and state estimates is theoretically difficult to prove.

In application of feedback control to physiological systems there will always be a compromise between the model complexity and the closed-loop performance. In our approach we intend to keep the model complexity as well as the identification procedure at a minimum, while achieving robust tracking performance. Our intention is to design a nonlinear controller based on the back-stepping method (Sepulchre et al., 1997) employing a simplified model of the knee-joint dynamics (Section 4.1). This control design approach possesses more inherent robustness with respect to modelling errors compared to other nonlinear control design approaches. Simulation results based on the control 
of a complex physiological model of the knee-joint will be given.

\section{Experimental Set-Up}

The experimental set-up for identification is depicted in Fig. 1. The subject is seated on a bed with the unloaded shank free to swing. Pulsewidth modulated electrical current pulses are sent to the knee extensor muscles via surface electrodes and cause contractions and knee-joint motion. The knee angle $y$ (system output) is measured and sampled every $50 \mathrm{~ms}$ by a 3D-motion analysis system (zebris Medizintechnik GmbH, 1999) working with ultrasound. It delivers the angle measurements in real-time (time delay of $70 \mathrm{~ms}$ ) via the parallel port to a laptop. A multi-channel stimulator (Phillips et al., 1993) is connected to the laptop via the serial port and delivers the current pulses with pulsewidth $p w$ up to $500 \mu \mathrm{s}$. During the experiments the pulsewidth serves as a variable input signal whereas the current amplitude $I=80 \mathrm{~mA}$ and the stimulation frequency $f=20 \mathrm{~Hz}$ are fixed.

The input signal during identification is chosen as a Multi-level Pseudo-Random Sequence (M-level PRS) (Godfrey, 1993) in order to excite the nonlinear system behaviour. An M-level PRS is a periodic, deterministic signal which has an autocorrelation function similar to white noise.

A 57 year-old T10 complete paraplegic male subject, 2 years post-injury, training his quadriceps muscles for a FES-cycling project, participated in this study. Informed consent was obtained from the subject.

\section{Identification of the Electrically Stimulated Quadriceps Muscle}

\subsection{Model Structure}

The shank-quadriceps dynamics are modelled as the interconnection of a passive part (Sec. 3.1.1), including the equation of motion and passive elastic/viscous joint properties, and an active part (Sec. 3.1.2), consisting of muscle activation and contraction dynamics. Passive muscle properties have been assigned to the joint in order to keep the number of muscle parameters small. The passive system part is nearly time-invariant, whereas the active part (muscle actuator) possesses a large day-to-day variation and is affected by muscle fatigue due to prolonged stimulation. 


\subsubsection{Passive Viscous-Elastic Characteristics and Equation of Motion.}

The total knee-joint moment is given by

$$
M_{K}=M_{e}+M_{g}+M_{v}+M_{a}
$$

with $M_{e}$ : passive elastic moment, $M_{g}$ : gravitational moment, $M_{v}$ : passive viscous moment and $M_{a}$ : active moment generated by quadriceps stimulation. The gravitational component is determined by

$$
M_{g}\left(x_{1}\right)=-m g l \sin \left(x_{1}-x_{1}^{*}\right)
$$

where $m$ is the mass of the shank-foot complex and $l$ is the distance between knee and centre of mass. The knee-joint angle $x_{1}$ as well as the angle $x_{1}^{*}$ are defined in Fig. 1. The elastic moment $M_{e}\left(x_{1}\right)$ is expressed as an exponential function as proposed and experimentally verified in Ferrarin and Pedotti (2000). Passive viscous joint properties have been modelled as a nonlinear damping function

$$
M_{v}\left(x_{2}\right)=B_{1} \tanh \left(-B_{2} x_{2}\right)-B_{3} x_{2}
$$

where $B_{1}, B_{2}$ and $B_{3}$ are positive constants and $x_{2}$ the angular velocity. Note that Coulombic friction effects are captured by this damping function. The Equation of Motion (EoM) is given by

$$
\dot{x}_{2}=\frac{1}{J_{K}} M_{K}\left(x_{1}, x_{2}, M_{a}\right)
$$

where $J_{K}$ represents the moment of inertia of the shank-foot complex about the knee-joint. The anthropometric parameters $m, J_{K}$ and $l$ are estimated by measuring the height and weight of the subject and using regression equations (Stein et al., 1996). A passive pendulum trial is performed to obtain stiffness and damping parameters. When no electrical stimulation is active, these parameters can be calculated by using a nonlinear least-squares algorithm applied to the model equations.

\subsubsection{Nonlinear Muscle Model}

The active muscle moment $M_{a}$ is determined by the product of an activation dynamics and a nonlinear static contraction function (cf. Fig. 2).

The activation dynamics consist of a static recruitment curve and a transfer function connected in series. The recruitment curve gives a measurement for the normalised amount of activated motor units depending on the pulsewidth $p w$. This measurement is labelled as stimulation level. A piecewise linear func- 
tion describes the recruitment curve:

$$
s t=\left\{\begin{array}{rlrl}
0, & p w<p w_{t h r} \\
\frac{p w-p w_{t h r}}{p w_{s a t}-p w_{t h r}}, & p w_{t h r} \leq p w & \leq p w_{s a t} \\
1, & p w & >p w_{s a t}
\end{array}\right.
$$

where $p w_{t h r}$ is the pulsewidth for which first motor units are recruited and $p w_{\text {sat }}$ stands for the pulsewidth where all motor units are recruited. These threshold and saturation levels can be determined as follows under non-isometric conditions: the pulsewidth for which the shank starts to move is taken as $p w_{t h r}$, and the pulsewidth for which the leg reaches full extension is approximately chosen as pwat.

The temporal behaviour of the muscle activation is mainly influenced by calcium $\mathrm{Ca}^{2+}$ release dynamics, finite conduction velocities in the membrane system and delays from the chemical reactions involved. To describe these effects a second order transfer function with an input time delay $T_{d}$ as proposed by Riener and Fuhr (1998) is used:

$$
\begin{aligned}
& \dot{x}_{3}=x_{4} \\
& \dot{x}_{4}=-2 w_{0} D x_{4}-w_{0}^{2} x_{3}+w_{0}^{2} s t\left(t-T_{d}\right)
\end{aligned}
$$

where the output $x_{3}$ is the normalised activation of the muscle, $w_{0}$ and $D=1$ are the undamped natural frequency and the damping factor of the calcium dynamics respectively.

Nonlinear Contraction Function. The active knee moment produced by the quadriceps is given by

$$
M_{a}=F_{m}\left(\boldsymbol{x}_{p}\right) x_{3}
$$

where $F_{m}[\mathrm{Nm}]$ is the nonlinear contraction function which defines the maximal possible active torque in the case of full muscle activation dependent on kneejoint angle and velocity which have been arranged in a vector $\boldsymbol{x}_{p}=\left[\begin{array}{ll}x_{1} & x_{2}\end{array}\right]^{\prime}$.

With the aim to have little constraints on the form of the nonlinear contraction function, a Normalised Radial Basis Function (NRBF) network (Nelles, 2001) is used to parameterise $F_{m}$ :

$$
F_{m}\left(\boldsymbol{x}_{p}\right)=\sum_{i=1}^{M} \psi_{i} \tilde{\varphi}_{i}\left(\left\|\boldsymbol{x}_{p}-\boldsymbol{c}_{i}\right\|_{\Sigma_{i}}\right)
$$

Here, the contraction function is described as the sum of $M$ normalised radial basis functions $\tilde{\varphi}_{i}, i=1, \ldots, M$ which are weighted by the constants $\psi_{i}, i=$ 
$1, \ldots, M$. The function $\tilde{\varphi}_{i}$ is given by

$$
\tilde{\varphi}_{i}\left(\left\|\boldsymbol{x}_{p}-\boldsymbol{c}_{i}\right\|_{\Sigma_{i}}\right)=\frac{\varphi_{i}\left(\left\|\boldsymbol{x}_{p}-\boldsymbol{c}_{i}\right\|_{\Sigma_{i}}\right)}{\sum_{l=1}^{M} \varphi_{l}\left(\left\|\boldsymbol{x}_{p}-\boldsymbol{c}_{l}\right\|_{\Sigma_{l}}\right)}
$$

where $\varphi_{i}$ is a non-normalised Radial Basis Function (RBF) chosen to be a Gaussian function:

$$
\varphi_{i}\left(\left\|\boldsymbol{x}_{p}-\boldsymbol{c}_{i}\right\|_{\Sigma_{i}}\right)=\exp \left(-1 / 2|| \boldsymbol{x}_{p}-\boldsymbol{c}_{i} \|_{\Sigma_{i}}^{2}\right) .
$$

The argument $\left\|\boldsymbol{x}_{p}-\boldsymbol{c}_{i}\right\|_{\Sigma_{i}}$ represents the distance of the NRBF network input vector $\boldsymbol{x}_{p}$ from the centre vector $\boldsymbol{c}_{i}$ and is calculated by

$$
\left\|\boldsymbol{x}_{p}-\boldsymbol{c}_{i}\right\|_{\Sigma_{i}}=\sqrt{\left(\boldsymbol{x}_{p}-\boldsymbol{c}_{i}\right)^{\prime} \boldsymbol{\Sigma}_{i}\left(\boldsymbol{x}_{p}-\boldsymbol{c}_{i}\right)}
$$

with $\boldsymbol{\Sigma}_{i}$ being a norm matrix. For this application the $\boldsymbol{\Sigma}_{i}$ matrix is chosen to be diagonal; so, it contains the inverse variances for each input dimension.

NRBF networks possess a good interpolation and extrapolation behaviour (constant) (Nelles, 2001). Furthermore it can be guaranteed that the NRBF output lies in the interval $\min \left(\psi_{i}\right) \leq F_{m} \leq \max \left(\psi_{i}\right), i=1, \ldots, M$. This is a result of the fact that the sum of all normalised basis functions $\tilde{\varphi}_{i}, i=1, \ldots, M$ is equal to 1 .

\subsubsection{State-Space Model Representation}

The shank-quadriceps dynamics can be compactly written as state-space model with the following state vector, input and output:

$$
\begin{aligned}
\boldsymbol{x} & =\left[\begin{array}{llll}
x_{1} & x_{2} & x_{3} & x_{4}
\end{array}\right]^{\prime} \\
y & =x_{1} \\
u & =p w\left(t-T_{d}\right) .
\end{aligned}
$$

Elements of the state vector $\boldsymbol{x}$ are joint angle and velocity as well as the states of the calcium dynamics. The knee-joint angle is defined as output, and the time delayed pulsewidth is taken as system input. The form of the state-space model is then

$$
\begin{aligned}
\dot{\boldsymbol{x}} & =\boldsymbol{f}(\boldsymbol{x}, u) \\
y & =\boldsymbol{c}^{\prime} \boldsymbol{x}
\end{aligned}
$$


with the vector function $\boldsymbol{f}$ given by

$$
\boldsymbol{f}(x, u)=\left[\begin{array}{l}
x_{2} \\
\frac{1}{J_{K}}\left[M_{e}\left(x_{1}\right)+M_{g}\left(x_{1}\right)+M_{v}\left(x_{2}\right)+F_{m}\left(\boldsymbol{x}_{p}\right) x_{3}\right] \\
x_{4} \\
-2 w_{0} D x_{4}-w_{0}^{2} x_{3}+w_{0}^{2} \operatorname{st}(u)
\end{array}\right]
$$

The vector $\boldsymbol{c}$ is defined as $\boldsymbol{c}=\left[\begin{array}{llll}1 & 0 & 0 & 0\end{array}\right]^{\prime}$.

\subsection{Extended Kalman Filter}

Online estimation of the state vector $\boldsymbol{x}$ and some muscle parameters is investigated. Muscle parameters, considered for online estimation, are the weights of the NRBF network which are arranged in a vector $\boldsymbol{\psi}=\left[\psi_{1}, \cdots, \psi_{M}\right]^{\prime}$, as well as the frequency $w_{0}$ of the activation dynamics.

All parameters to be tracked form a parameter vector $\boldsymbol{\theta}$ :

$$
\boldsymbol{\theta}=\left[\begin{array}{ll}
w_{0} & \boldsymbol{\psi}^{\prime}
\end{array}\right]^{\prime} .
$$

A common approach in parameter estimation is to model all the parameters by a differential equation. By setting

$$
\dot{\theta}=\mathbf{0}
$$

we assume that model parameters are time-invariant or slowly varying in comparison to the process dynamics. Equation (20) is added to the plant statespace model and a nonlinear observer is then applied to estimate the metastate $\boldsymbol{z}=\left[\begin{array}{ll}\boldsymbol{x}^{\prime} & \boldsymbol{\theta}^{\prime}\end{array}\right]^{\prime}$. The resulting dynamical system for the meta-state is given by

$$
\begin{aligned}
\dot{\boldsymbol{z}}=\left[\begin{array}{c}
\dot{\boldsymbol{x}} \\
\dot{\boldsymbol{\theta}}
\end{array}\right] & =\underbrace{\left[\begin{array}{l}
\boldsymbol{f}(\boldsymbol{x}, u, \boldsymbol{\theta}) \\
\mathbf{0}
\end{array}\right]}_{\boldsymbol{f}^{z}(\boldsymbol{z}, u)} \\
y & =\underbrace{\left[\begin{array}{ll}
\boldsymbol{c}^{\prime} & \mathbf{0}
\end{array}\right]}_{\boldsymbol{c}^{z^{\prime}}}\left[\begin{array}{l}
\boldsymbol{x} \\
\boldsymbol{\theta}
\end{array}\right] .
\end{aligned}
$$

The estimation of the meta-state is carried out using an Extended Kalman Filter (EKF) (Jazwinski, 1970) under following conditions and assumptions:

- Only discrete-time noisy angle measurements are available as observations (sample time $T_{s}=50 \mathrm{~ms}$ ). 
- The input signal is constant between two pulses: $u(t)=u_{k}=u\left(k T_{s}\right), \quad k T_{s} \leq$ $t<(k+1) T_{s}$.

- Estimates $\hat{\boldsymbol{z}}_{k}=\hat{\boldsymbol{z}}\left(k T_{s}\right)$ of the meta-state are required at discrete-time instants only.

To perform this estimation task, we assume that a discrete-time state-space model of the shank-quadriceps dynamics is available, that describes the system (21-22) exactly at the time instants $k T_{s}$. The discrete-time model has the following form:

$$
\begin{aligned}
& \boldsymbol{z}_{k}=\boldsymbol{f}_{s}^{z}\left(\boldsymbol{z}_{k-1}, u_{k-1}\right)+\boldsymbol{v}_{k-1} \\
& y_{k}=\boldsymbol{c}^{z^{\prime}} \boldsymbol{z}_{k}+r_{k}
\end{aligned}
$$

where $\boldsymbol{f}_{s}^{z}$ is an appropriate transition function. Measurement noise $r_{k}$ and process noise $\boldsymbol{v}_{k}$ are uncorrelated sampled white noise signals with covariance $\mathcal{E}\left(r_{n} r_{k}\right)=\delta_{n, k} R_{k}$ and covariance matrix $\mathcal{E}\left(\boldsymbol{v}_{n} \boldsymbol{v}_{k}^{\prime}\right)=\delta_{n, k} \boldsymbol{V}_{k}$ respectively. Here, $\mathcal{E}$ is the expectation operator and $\delta$ is the Kronecker symbol.

The EKF provides an efficient method for generating approximate maximumlikelihood estimates of the meta-state of the discrete-time system (23-24). The filter optimally combines noisy observations with predictions from the known dynamical model and involves the following recursive procedure (Friedland, 1996):

\section{1st step: Propagate the state}

$$
\tilde{\boldsymbol{z}}_{k}=\boldsymbol{f}_{s}^{z}\left(\hat{\boldsymbol{z}}_{k-1}, u_{k-1}\right)
$$

Here, $\tilde{\boldsymbol{z}}_{k}$ is the a priori state estimate, and represents the state estimate immediately before the $k$ th observation of the knee-joint angle. This estimate is based on the estimate $\hat{\boldsymbol{z}}_{k-1}$ which is the state estimate after the $(k-1)$ th observation of the previous time step. The state estimate immediately after an observation is called a posteriori estimate.

As the transition matrix $\boldsymbol{f}_{s}^{z}$ is not known explicitly, it will be defined implicitly as the solution of the nonlinear differential equation (21). The estimate $\tilde{\boldsymbol{z}}_{k}$ is the result of numerical integration of (21) over the interval $(k-1) T_{s} \leq t \leq k T_{s}$ (during which the control signal $u(t)=u_{k-1}$ is constant) starting with the initial condition $\boldsymbol{z}\left((k-1) T_{s}\right)=\hat{\boldsymbol{z}}_{k-1}$.

\section{2nd step: Propagate the error covariance matrix}

The a priori (before observation) estimation error covariance matrix $\tilde{\boldsymbol{P}}_{k}$ is updated by

$$
\tilde{\boldsymbol{P}}_{k}=\boldsymbol{\Phi}_{k-1} \hat{\boldsymbol{P}}_{k-1} \boldsymbol{\Phi}_{k-1}^{\prime}+\boldsymbol{V}_{k}
$$


where the matrix $\boldsymbol{\Phi}_{k-1}$ is the Jacobian matrix of the transition function $\boldsymbol{f}_{s}^{z}$ :

$$
\boldsymbol{\Phi}_{k-1}=\left.\frac{\partial \boldsymbol{f}_{s}^{z}\left(\boldsymbol{z}_{k-1}, u_{k-1}\right)}{\partial \boldsymbol{z}_{k-1}}\right|_{\boldsymbol{z}_{k-1}=\hat{\boldsymbol{z}}_{k-1}}
$$

As the transition function $\boldsymbol{f}_{s}^{z}$ is not explicitly given, the matrix $\boldsymbol{\Phi}_{k-1}$ is calculated by numerical integration of the linearised matrix equation

$$
\dot{\boldsymbol{\Phi}}=\boldsymbol{A}(t) \boldsymbol{\Phi}
$$

over the interval $(k-1) T_{s} \leq t \leq k T_{s}$ starting with initial conditions $\boldsymbol{\Phi}((k-$ 1) $\left.T_{s}\right)=\boldsymbol{I}$ and with $\boldsymbol{A}(t)$ being the Jacobian matrix of the nonlinear function $\boldsymbol{f}^{z}\left(\boldsymbol{z}, u_{k-1}\right)$ :

$$
\boldsymbol{A}(t)=\left.\frac{\partial \boldsymbol{f}^{z}\left(\boldsymbol{z}, u_{k-1}\right)}{\partial \boldsymbol{z}}\right|_{\boldsymbol{z}(t)}
$$

The meaning of the subscript $\boldsymbol{z}(t)$ is that the partial derivatives are computed along the trajectories in state-space defined by numerical integration of the continuous-time process equation (21).

\section{3rd step: Update the Kalman gain}

The current Kalman gain is computed from the a priori error covariance matrix $\tilde{\boldsymbol{P}}_{k}$ as follows:

$$
\boldsymbol{K}_{k}=\tilde{\boldsymbol{P}}_{k} \boldsymbol{c}^{\boldsymbol{z}}\left(\boldsymbol{c}^{\boldsymbol{z}} \tilde{\boldsymbol{P}}_{k} \boldsymbol{c}^{\boldsymbol{z}}+R_{k}\right)^{-1} .
$$

\section{4th step: State estimation update}

The a priori state estimate is corrected by

$$
\hat{\boldsymbol{z}}_{k}=\tilde{\boldsymbol{z}}_{k}+\boldsymbol{K}_{k}\left(y_{k}-\boldsymbol{c}^{\boldsymbol{z} \prime} \tilde{\boldsymbol{z}}_{k}\right)
$$

using the computed Kalman gain and the current observation $y_{k}$ in order to obtain the a posteriori state estimate $\hat{\boldsymbol{z}}(k)$.

\section{5th step: Update the error covariance}

Finally, the a posteriori error covariance is given by

$$
\hat{\boldsymbol{P}}_{k}=\left(\boldsymbol{I}-\boldsymbol{K}_{k} \boldsymbol{c}^{\boldsymbol{z} \prime}\right) \tilde{\boldsymbol{P}}_{k} .
$$

Note that the terms a priori and a posteriori for the estimation error covariance matrix depend only on the observation times and not on the observation data.

The initial approximate error covariance matrix $\hat{P}(0)$ for the EKF is chosen to be a diagonal matrix; the scalar diagonal elements of $\hat{P}(0)$ have the following values: 10 if related to plant state estimates, 1000 if related to parameter 
estimates of recruitment curve and calcium dynamics and 10000 if related to NRBF network weights. Larger values indicate that there is a larger discrepancy between initialisation and real values.

Low level state noise is assumed for the states which represent parameters. This prevents the gain matrix of the EKF from becoming too small. As a consequence, changes in the muscle parameters can be quickly detected also when the Kalman filter is running for a long time. The noise related to the states $x_{2}$ and $x_{4}$ is modelled with higher variance since large unpredictable variations (disturbances) in the neural activation and the knee moment are likely. The noise covariance $R_{k}$ is set accordingly to the specifications of the measurement system.

\subsection{Identification Results}

The extended Kalman filter was tested in simulations and with real data. To describe the function $F_{m}$, nine RBFs $(M=9)$ were used whereas centres of the Gaussian functions are chosen as the points of a regular grid; these points cover the interesting angle-velocity region during the non-isometric muscle contractions. Variances of all non-normalised RBFs are all equal. Significant overlapping of the non-normalised RBFs was selected to guarantee a high smoothness of the resulting nonlinear contraction function $F_{m}$. The elements of the meta-state vector were initialised as follows:

$$
\hat{x}_{1}(0)=y(0), \hat{x}_{2}(0)=0, \hat{x}_{3}(0)=0 \text {, and } \hat{x}_{4}(0)=0 .
$$

Initial estimates $\hat{\psi}_{i}(0), i=1, \ldots, M$, for the NRBF network weights were randomly chosen from the range $[-0.1,0.1]$.

\subsubsection{Simulations using a Plant Simulator}

The proposed model structure and EKF have been tested in simulations first. A complex model of the lower limbs served as a substitution for the real plant. This model was developed by Riener and Fuhr (1998), and will be called plant simulator in the following.

Two individual sub muscle groups of the quadriceps muscle group, namely rectus femoris and vasti, have been modelled independently with their own activation and contraction dynamics. A Hill-type muscle model is assumed in each contraction dynamics. The product of muscle activation and maximal isometric force is scaled by a nonlinear force - muscle length relation and force - muscle contraction velocity relation. Muscle lengths and contraction velocities are parameterised by the joint angle and angular velocity of the 
knee which the muscles span via moment arms. The moment arms (defined as the derivatives of the muscle lengths with respect to joint angle) are derived under the assumption, that the knee is not a simple hinge joint. Active joint moments are given by the sum of moments produced by each muscle group, i.e. muscle force multiplied by moment arm.

EoM as well as passive viscous and elastic joint properties are identical to the description given in Sec. 3.1.1.

The EKF approach was evaluated in simulations with the plant simulator serving as real plant; output measurement noise with a variance of $0.0002 \mathrm{rad}^{2}$ was introduced. Fig. 3 shows the knee-joint angle (plant simulator output) as well as the corresponding stimulation pulsewidth. The a priori angle estimate of the EKF is also shown in this plot. However, because of the close fit it is not possible to distinguish both signals visually. In Fig. 4, the active moment inside the plant simulator is plotted together with the a priori estimate of the EKF. Already after approximately $5 \mathrm{~s}$ one can recognise a significant reduction in prediction error.

The corresponding parameter estimates of the muscle dynamics are depicted in Fig. 5; the upper graph shows the activation dynamics frequency $\hat{w}_{0}$ while elements of the estimated vector $\hat{\boldsymbol{\psi}}$ are shown in the lower graph. The initial estimate for the frequency was chosen as $\hat{w}_{0}(0)=201 / \mathrm{s}(251 / \mathrm{s}$ for rectus femoris and $331 / \mathrm{s}$ for vasti within the plant simulator). The EKF converges steadily within approximately $30 \mathrm{~s}$. The estimated nonlinear contraction function $\hat{F}_{m}$ after 100 s identification is shown in Fig. 6 .

The estimated model after $100 \mathrm{~s}$ identification was further used to simulate the plant behaviour. For this test, the completely estimated model is only driven by the input signal while online parameter identification and state estimation are switched off. The result of this simulation test is given in Fig. 7.

\subsubsection{Evaluation using Experimental Data}

The EKF approach was further evaluated in experiments with one paraplegic male subject. Fig. 8 shows an applied stimulation sequence and the corresponding measured output signal (angle) as well as the a priori angle estimate of the EKF. The corresponding parameter estimates of the muscle dynamics are depicted in Fig. 9; the upper graph shows the parameter $\hat{w}_{0}$ of the activation dynamics while elements of the estimated vector $\hat{\boldsymbol{\psi}}$ are shown in the lower graph.

The nonlinear recruitment curve was experimentally determined as described in Sec. 3.1.2. The initial estimate for the frequency $w_{0}$ was taken from literature. The time delay $T_{d}$ was estimated by correlation analysis of recorded 
input-output data of the system.

The estimated model after $80 \mathrm{~s}$ identification was further used to simulate the plant behaviour for the time range 80-105 s. For this test, the model is only driven by the input signal while online muscle identification and state estimation are switched off. The result of this simulation test is given in Fig. 10.

The EKF converges steadily. The one step ahead angle prediction of the EKF (a priori estimate) is fitting very well to the measured angle. However, the simulation test shows some discrepancies between model output and measured angle. This may be the effect of choosing a too simple model structure. Note that no enhanced optimisation of the NRBF network structure (no. of NRBF etc.) was carried out. Other reasons for the slight discrepancies may be spinal reflexes (Nickolls et al., 2004) which are not described by our model. Also, the estimation of the passive model part by means of simple pendulum tests may not have given the best estimates for elastic and viscous passive moment characteristics.

\section{Nonlinear Controller Design}

The system identification approach outlined before is subsequently used to identify a simplified model of the quadriceps muscle. This model will be employed for nonlinear controller design. A simulation study based on the plant simulator introduced in Section 3.3.1 is carried out to validate closed-loop performance.

\subsection{Simplified Model for Control Design}

As the proposed model in Section 3.1 is too complex to be used in a control scheme, a simplified model will be derived. A high accuracy of the model is not required for a robust controller design. The following model is used for identification and for control design purposes:

$$
\begin{aligned}
\dot{x_{1}} & =x_{2} \\
J \dot{x_{2}} & =M_{g}\left(x_{1}\right)+M_{e}\left(x_{1}\right)+M_{v}\left(x_{2}\right)+F_{m}\left(x_{1}, x_{2}\right) x_{3} \\
\dot{x}_{3} & =a x_{3}+b u .
\end{aligned}
$$

Here, $x_{1}, x_{2}$ and $x_{3}$ represent respectively the angle, the angular velocity and the muscle activation level. $F_{m}\left(x_{1}, x_{2}\right)$ is a linear contraction function which defines the maximum possible active torque in the case of full muscle activation dependent on the knee-joint angle and the velocity. This relationship is 
modelled as a linear function of the knee-joint angle and the velocity

$$
F_{m}\left(x_{1}, x_{2}\right)=d x_{1}+e x_{2}+f
$$

The control signal $u[0-1]$ represents the normalised and de-saturated stimulation level. The level $u=0$ corresponds to the stimulation pulse-width value where the muscle starts to contract, whereas $u=1$ corresponds to the pulsewidth value for which all motor units are recruited by the stimulation, i.e. the muscle is fully contracted. The parameter $J_{K}$ is the moment of inertia of the foot-shank complex. $M_{g}=-m g l \sin \left(x_{1}-x_{1}^{*}\right)$ represents the gravitational component, where $m$ is the mass of the foot-shank complex and $l$ is the distance between the knee and the centre of the mass, and $g$ is the gravitational constant. The elastic moment is defined by

$$
M_{e}\left(x_{1}\right)=-k_{1} \exp \left(-k_{2} x_{1}\right)\left(x_{1}-k_{3}\right)
$$

where $k_{1}, k_{2}$ and $k_{3}$ are positive coefficients. Viscous joint properties are modelled as a linear damping: $M_{v}=-B x_{2}$, where $B$ is the damping constant.

No complex model of the muscle activation is considered. The activation dynamics are modelled as first order linear dynamics, with the parameters $a$ and $b$.

\subsection{Identification of the Simplified Model}

The anthropometric parameters $m, J_{K}$ and $l$ were directly collected from the plant simulator (Riener and Fuhr, 1998) for this simulation case study. Then in a simulation, a passive pendulum test was performed in order to obtain stiffness and damping parameters. When no electrical stimulation is active, these parameters can be calculated by using a nonlinear least-squares algorithm applied to the model equations (33-35).

In order to identify the parameters in the simplified contraction function and the parameters in the activation dynamics, the EKF described in Section 3 was used. The contraction function was simplified to be linear in position and velocity. This choice is acceptable for controller design and gives a good approximation of the NRBF network in the interesting range of positions and velocities for the plant simulator. The number of parameters to describe $F_{m}$ was reduced by this from nine to three. The activation dynamics are also approximated to be a first order system, and a time delay was not considered in the identification.

The model parameters used in simulations and for controller design are listed in Table 1. An m-level signal was used as input signal during the EKF identification. All parameters converged to steady state value by $50 \mathrm{~s}$. 


\subsection{Backstepping Control}

A nonlinear controller is designed for tracking the knee-joint angle based on the back-stepping technique in Sepulchre et al. (1997) using the simplified model of the knee-joint (33-35). The main argument for applying the backstepping technique instead of other nonlinear controller design techniques is the inherent robustness of the resulting controller. A state transformation is introduced in order to reformulate the tracking problem into a stabilisation problem. The new states are defined by

$$
\begin{aligned}
& \xi_{1}=x_{1}-x_{d} \\
& \xi_{2}=x_{2} \\
& \xi_{3}=x_{3} .
\end{aligned}
$$

Here, $\xi_{1}$ is the tracking error, and $x_{d}$ represents the reference angle to be tracked.

By applying this state transformation, the new state-space model becomes

$$
\begin{aligned}
& \dot{\xi}_{1}=\xi_{2}-\dot{x}_{d} \\
& \dot{\xi}_{2}=\frac{1}{J_{K}}\left(M_{g}\left(\xi_{1}+x_{d}\right)+M_{e}\left(\xi_{1}+x_{d}\right)+M_{v}\left(\xi_{2}\right)\right)+\frac{1}{J_{K}} F_{m}\left(\xi_{1}+x_{d}, \xi_{2}\right) \xi_{3} \\
& \dot{\xi}_{3}=a \xi_{3}+b u
\end{aligned}
$$

This system possesses an important structure, it is in a strict feedback form. This means that except for the integrator chain, all other interconnections in the system are feedback interconnections. For this class of systems the backstepping technique can be applied, which offers a constructive way to stabilise a system. The back-stepping design technique is working recursively. As a first step, a part of the plant is stabilised. Then this subsystem is augmented by the next system equation and a new stabilising controller is found using the already determined control law designed for the previous subsystem in an efficient way.

We are starting by looking at the first subsystem $\Sigma_{1}$ consisting of the state equation for $\xi_{1}$ :

$$
\Sigma_{1}: \dot{\xi}_{1}=\xi_{2}-\dot{x}_{d}
$$

This system can be stabilised by using $\xi_{2}=\alpha_{1}\left(\xi_{1}\right)$ as a virtual control law. We are now defining an output which we want to regulate to zero. This output is chosen to be the first state $z_{1}=\xi_{1}$. A Control Lyapunov Function (CLF) $V_{1}$ 
is chosen as

$$
V_{1}=\frac{1}{2} z_{1}^{2}
$$

whose time derivative is

$$
\begin{aligned}
\dot{V}_{1} & =z_{1} \dot{z}_{1} \\
& =\left(z_{1}\right)\left(-\dot{x}_{d}+\alpha_{1}\left(\xi_{1}\right)\right) .
\end{aligned}
$$

The virtual control law $\alpha_{1}\left(\xi_{1}\right)$ is now chosen to make the time derivative $\dot{V}_{1}<0$. From Lyapunov stability theory we know, that such a control law is stabilising the first subsystem. Taking

$$
\alpha_{1}\left(\xi_{1}\right)=\dot{x}_{d}-K_{1} z_{1}
$$

where $K_{1}>0$ is a tuning parameter yields the CLF derivative

$$
\dot{V}_{1}=-K_{1} z_{1}^{2} \text {. }
$$

Hence, the first subsystem is successfully stabilised by the virtual control law $\alpha_{1}\left(\xi_{1}\right)$ since the derivative $\dot{V}_{1}$ is negative along the trajectories of this subsystem. We are now extending the system to the subsystem $\Sigma_{2}$ given by

$$
\Sigma_{2}:\left\{\begin{array}{l}
\dot{\xi}_{1}=\xi_{2}-\dot{x}_{d} \\
\dot{\xi}_{2}=\frac{1}{J_{K}}\left(M_{g}\left(\xi_{1}+x_{d}\right)+M_{e}\left(\xi_{1}+x_{d}\right)+M_{v}\left(\xi_{2}\right)\right)+\frac{1}{J_{K}} F_{m}\left(\xi_{1}+x_{d}, \xi_{2}\right) \xi_{3} .
\end{array}\right.
$$

In order to exploit the fact that we found a stabilising controller for the first subsystem, we rewrite the system by introducing a change in variables $z_{2}=$ $\xi_{2}-\alpha_{1}\left(\xi_{1}\right)$.

$$
\Sigma_{2}:\left\{\begin{array}{l}
\dot{\xi}_{1}=-\dot{x}_{d}+\alpha_{1}\left(\xi_{1}\right)+z_{2} \\
\dot{\xi}_{2}=\frac{1}{J_{K}}\left[M_{g}\left(\xi_{1}+x_{d}\right)+M_{e}\left(\xi_{1}+x_{d}\right)+M_{v}\left(\xi_{2}\right)\right]+\frac{1}{J_{K}} F_{m}\left(\xi_{1}+x_{d}, \xi_{2}\right) \xi_{3}
\end{array}\right.
$$

The subsystem $\Sigma_{1}$ has now an asymptotic stable origin when $z_{2}$ is zero. By introducing $\xi_{3}=\alpha_{2}\left(\xi_{1}, \xi_{2}\right)$ as a virtual control input, we can stabilise this system. The following CLF is used to stabilise the system $\Sigma_{2}$

$$
V_{2}=V_{1}+\frac{1}{2} J_{K} z_{2}^{2}
$$

with the derivative

$$
\begin{aligned}
\dot{V}_{2} & =-K_{1} z_{1}^{2}+z_{1} z_{2}+z_{2} \dot{z}_{2} \\
& =-K_{1} z_{1}^{2}+z_{1} z_{2}+z_{2}\left(M_{g}\left(\xi_{1}+x_{d}\right)+M_{e}\left(\xi_{1}+x_{d}\right)-B z_{2}-B \alpha_{1}+F_{m} \alpha_{2}-J_{K} \dot{\alpha}_{1}\right) .
\end{aligned}
$$


The stabilising virtual control law $\alpha_{2}\left(\xi_{1}, \xi_{2}\right)$ is taken as

$$
\begin{aligned}
\alpha_{2}\left(\xi_{1}, \xi_{2}\right)= & \frac{1}{F_{m}}\left(-z_{1}+B \alpha_{1}-K_{2} z_{2}-\right. \\
& \left.M_{g}\left(\xi_{1}+x_{d}\right)-M_{e}\left(\xi_{1}+x_{d}\right)+J_{K} \dot{\alpha}_{1}\right)
\end{aligned}
$$

where $K_{2}>0$ is a tuning parameter. Hence, $\dot{V}_{2}$ becomes

$$
\dot{V}_{2}=-K_{1} z_{1}^{2}-\left(K_{2}+B\right) z_{2}^{2}<0 .
$$

Then, we are extending the second subsystem with the last state equation of (41-43). This gives the following system

$$
\Sigma_{3}:\left\{\begin{aligned}
\dot{\xi}_{1}= & \xi_{2}-\dot{x}_{d} \\
\dot{\xi}_{2}= & \frac{1}{J_{K}}\left(M_{g}\left(\xi_{1}+x_{d}\right)+M_{e}\left(\xi_{1}+x_{d}\right)\right. \\
& \left.+M_{v}\left(\xi_{2}\right)\right)+\frac{1}{J_{K}} F_{m}\left(\xi_{1}+x_{d}, \xi_{2}\right) \xi_{3} \\
\dot{\xi}_{3}= & a \xi_{3}+b u .
\end{aligned}\right.
$$

Again, we are introducing a new change in variables $z_{3}=\xi_{3}-\alpha_{2}\left(\xi_{1}, \xi_{2}\right)$, and achieving this system

$$
\Sigma_{3}:\left\{\begin{aligned}
\dot{\xi}_{1}= & \xi_{2}-\dot{x}_{d} \\
\dot{\xi}_{2}= & \frac{1}{J_{K}}\left(M_{g}\left(\xi_{1}+x_{d}\right)+M_{e}\left(\xi_{1}+x_{d}\right)\right. \\
& \left.+M_{v}\left(\xi_{2}\right)\right)+\frac{1}{J_{K}} F_{m}\left(\xi_{1}+x_{d}, \xi_{2}\right) \alpha_{2} \\
& +\frac{1}{J_{K}} F_{m}\left(\xi_{3}+x_{d}, \xi_{2}\right) z_{3} \\
\dot{\xi}_{3}= & a \xi_{3}+b u .
\end{aligned}\right.
$$

The subsystem $\Sigma_{2}$ is asymptotically stable when $z_{3}$ is zero. We are exploiting this fact by defining a new CLF for the total system.

$$
V_{3}=V_{2}+\frac{1}{2 \gamma} z_{3}^{2}
$$

where $\gamma$ is a tuning parameter introduced to shape the CLF. Differentiation yields

$$
\begin{aligned}
\dot{V}_{3} & =-K_{1} z_{1}^{2}-\left(K_{2}+B\right) z_{2}^{2}+F_{m} z_{2} z_{3}+z_{3} \dot{z}_{3} \\
& =-K_{1} z_{1}^{2}-\left(K_{2}+B\right) z_{2}^{2}+\frac{z_{3}}{\gamma}\left(a \xi_{3}+b u-\dot{\alpha}_{2}+\gamma F_{m} z_{2}\right) .
\end{aligned}
$$

We are now choosing the final control law to stabilise the overall system

$$
u=\frac{1}{b}\left(-\gamma F_{m} z_{2}-K_{3} z_{3}-a z_{3}-a \alpha_{2}+\dot{\alpha}_{2}\right)
$$


where $K_{3}>0$ is a tuning parameter which yields the derivative of the CLF for the total system:

$$
\dot{V}_{3}=-K_{1} z_{1}^{2}-\left(K_{2}+B\right) z_{2}^{2}-\frac{K_{3}}{\gamma} z_{3}^{2}
$$

Now when the control law is defined, it is straight forward to show that the equilibrium point $(\mathbf{z}=\mathbf{0})$ is asymptotically stable by applying Lyapunov stability theory (Khalil, 1996).

\subsubsection{Extention to Integral Action}

In order to compensate for modelling errors and static offset in the model, an integral action is implemented. Such a controller enables also the rejection of stepwise constant and slowly varying disturbances like fatigue.

A new state $\xi_{0}$ is introduced, which is the weighted integral of the tracking error

$$
\xi_{0}=\lambda \int_{0}^{t} \xi_{1} \mathrm{~d} \tau
$$

Now, by redefining the first virtual output to be a linear combination of the first two states

$$
z_{1}=\xi_{0}+\xi_{1}
$$

and reapplying the back-stepping procedure to this modified system, a control law with integral action is achieved. This controller was used in all the simulations described in this paper.

\subsection{Control Results}

In all simulations $20 \mathrm{~Hz}$ stimulation frequency is applied, as an implication the control signal was sampled every $50 \mathrm{~ms}$ and a zero order hold was applied. Generally, tracking performance in angle ranges up to 150 degree was very good with the designed controller. Above 150 degree the control performance was decreasing. In order to show the performance and robustness two different simulation tests were performed. First a sinusoidal reference signal $x_{d}$ varying between 110 and 150 degree, with a frequency of $0.5 \mathrm{~Hz}$ was used. The result of this test is shown in Figure 11. The knee-joint angle follows the reference closely with a small phase shift.

The next tests evaluated the robustness of the closed-loop system with respect to changes in the isometric maximal force of the plant simulator. The 
maximum isometric muscle force in the physiological model was changed for M. rectus femoris and M. vasti simultaneously to 50 and 150 percent of its nominal value. In Fig. 12 the tracking of a square wave signal is shown for the nominal case as well as for the altered plant. It can be observed that tracking performance is still good when the maximum muscle force is set to 150 percent of the nominal force. The closed-loop rise time is slightly increased when the maximum muscle force is assumed to be 50 percent of its nominal value, but tracking performance is still acceptable.

\section{Conclusion}

The quadriceps dynamics can be accurately described by the proposed model structure (modified Hill-type model). An Extended Kalman Filter represents a good tool to estimate the model parameters and gives state estimates of the quadriceps-shank dynamic at the same time. A good convergence of the estimation could be shown in simulations and real experiments.

As only discrete-time joint angle measurements are required for the parameter and state estimation this method is appealing for use in a clinical set-up; it is simple and no expensive sensors for velocity and acceleration measurements are required. However, the algorithm is computational expensive, but computational power of standard processors is growing steadily, and the real-time implementation of the algorithm is feasible.

By employing a simplified muscle model a nonlinear controller for knee-joint tracking was designed based on the back-stepping method. The designed controller showed a good performance at low angle ranges (90-150) degree. At higher angle ranges the performance was decreasing due to model simplifications. The decreasing performance at higher angle ranges is probably due to increasing misfit of model and real plant. We expect to observe this closed-loop behaviour in real experiments with paraplegic subjects.

The robustness tests have shown that the controller counteracts day-to-day variations of the maximum isometric muscle force, which can easily occur due to different electrode placement. The robustness tests give the indication that the controller can work without performing the identification procedure new at each session. But real experiments with subjects have to be carried out in order to verify this hypothesis.

The tuning of the four controller parameters requires a trial and error procedure, which only has to be carried out once. 
The integration of the EKF in nonlinear adaptive strategies for control of the paralysed lower limbs by means of FES is planned.

\section{References}

Chang, G.-C., Luh, J.-J., Liao, G.-D., Lai, J.-S., Cheng, C.-H., Kuo, B.-L., Kuo, T.-S., Mar. 1997. A Neuro-Control System for the Knee Joint Position Control with Quadriceps Stimulation. IEEE Trans. on Rehabilitation Engineering 5 (1), 2-11.

Chizeck, H. J., Chang, S., Stein, R. B., Scheiner, A., Ferencz, D. C., Jan. 1999. Identification of electrically stimulated quadriceps muscles in paraplegic subjects. IEEE Trans. on Biomedical Engineering 46 (1), 51-61.

Ferrarin, M., Palazzo, F., Riener, R., Quientern, J., Sep. 2001. Model-Based Control of FES-Induced Single Joint Movements. IEEE Trans. on Neural Systems and Rehabilitation Engineering 9 (3), 245-257.

Ferrarin, M., Pedotti, A., Sep. 2000. The relationship between electrical stimulus and joint torque: A dynamic model. IEEE Trans. on Rehabilitation Engineering 8 (3), 342-352.

Franken, H. M., Veltink, P. H., Tijsmans, R., Nijmeier, H., Boom, H. B. K., Jun. 1995. Identification of Quadriceps-Shank Dynamics Using Randomized Interpulse Interval Stimulation. IEEE Trans. on Rehabilitation Engineering 3 (2), 182-190.

Friedland, B., 1996. Advanced Control System Design. Prentice-Hall International, Inc.

Godfrey, K., 1993. Perturbation Signals for System Identification. Prentice Hall.

Gollee, H., Hunt, K. J., Jun. 1997. Nonlinear Modelling and Control of Electrically Stimulated Muscle: A Local Model Network Approach. Int. J. of Control 68 (6), 1259-1288.

Hill, A., 1938. The heat of shortening and the dynamic constants of muscle. Proc. Roy. Soc. Lond. B136, 136-195.

Hill, A., 1970. First and Last Experiments in Muscle Mechanics. Cambridge University Press, London.

Jazwinski, A., 1970. Stochastic Processes and Filtering Therorie. Academic Press, New York.

Jezernik, S., Wassink, R., Keller, T., 2004. Sliding Mode Closed-Loop Control of FES: Controlling the Shank Movement. IEEE Trans. on Biomedical Engineering 51 (2), 263-272.

Khalil, H. K., 1996. Nonlinear Systems. Prentice-Hall.

Nelles, O., 2001. Nonlinear System Identification. Springer.

Nickolls, P., Collins, D., Gorman, R., Burke, D., Gandevia, S., 2004. Forces consistent with plateau-like behaviour of spinal neurons evoked in patients with spinal cord injuries. Brain 127, 660-670. 
Phillips, G., Adler, J., Taylor, S., 1993. A portable programmable stimulator for surface FES. In: Proceedings of Llubljana FES Conference. Ljubljana, Slovenia, pp. 166-168.

Popović, D., Sinkjær, T., 2000. Control of Movement for the Physically Disabled. Springer.

Previdi, F., 2002. Identifcation of black-box nonlinear models for lower limb movement control using functional electrical stimulation . Control Engineering Practice 10, 91-99.

Previdi, F., Carpanzano, E., 2003. Design of a gain scheduling controller for knee-joint angle control by using functional electrical stimulation. IEEE Trans. on Control Systems Technology 11, 310-324.

Previdi, F., Schauer, T., Savaresi, S., Hunt, K., 2004. Data-Driven Control Design for Neuroprotheses: A Virtual Reference Feedback Tuning (VRFT) Approach . IEEE Trans. on Control Systems Technology 12 (1), 176-182.

Riener, R., Fuhr, T., Jun. 1998. Patient-Driven Control of FES-Supported Standing Up: A Simulation Study. IEEE Trans. on Rehabilitation Engineering 6 (2), 113-124.

Riess, J., Abbas, J., Mar. 2000. Adaptive Neural Network Control of Cyclic Movements Using Functional Neuromuscular Stimulation. IEEE Trans. on Rehabilitation Engineering 8 (1), 42-52.

Schauer, T., Holderbaum, W., Hunt, K., June 2002. Sliding-Mode Control of Knee-Joint Angle: Experimental Results. In: Proc. of the 7th Annual Conference of the International Functional Electrical Stimulation Society (IFESS 2002). Ljubljana, Slovenia, pp. 316-318.

Schauer, T., Hunt, K., March-April 2000. Linear Modelling and Controller Design for the Single Limb Movement of Paraplegics using FES. In: Carson, E., Salzsieder, E. (Eds.), Modelling and Control Biomedical Systems 2000 (Including Biological Systems): A Proceedings Volume from the 4th IFAC Symposium. Elsevier Science Ltd, Karlsburg/Greifswald, Germany, pp. 712.

Schauer, T., Hunt, K., Fraser, M., Stewart, W., Previdi, F., August 2001. Identification of a Biomechanical System using Neural Networks. In: Bittanti, S. (Ed.), Adaptation and Learning in Control and Signal Processing 2001: A Proceedings Volume from the IFAC Workshop. Elsevier Science Ltd, Cernobbio-Como, Italy, pp. 49-56.

Sepulchre, R., Janković, M., Kokotović, P., 1997. Constructive Nonlinear Control. Springer.

Stein, R. B., Zehr, E. P., Lebiedowska, M. K., Popovic, D. B., Scheiner, A., Chizeck, H. J., Sep. 1996. Estimating mechanical parameters of leg segments in individuals with and without physical disabilities. IEEE Trans. on Rehabilitation Engineering 4 (3), 201-211.

zebris Medizintechnik GmbH, 1999. Measuring System for 3D-Motion Analysis. Isny im Allgäu, Germany. 


\section{Tables and Figures}

Table 1

Parameters for the simplified model used for controller design.

\begin{tabular}{cccccc}
\hline$m[\mathrm{~kg}]$ & $l[\mathrm{~m}]$ & $J_{K}\left[\mathrm{kgm}^{2}\right]$ & $B\left[\frac{\mathrm{Nms}}{\mathrm{rad}}\right]$ & $k_{1}\left[\frac{\mathrm{Nm}}{\mathrm{rad}}\right]$ & $k_{2}\left[\frac{1}{\mathrm{rad}}\right]$ \\
\hline 4.3 & 0.28 & 0.39 & 0.6 & 7.9 & 1.68
\end{tabular}

\begin{tabular}{cccccc}
\hline$k_{3}[\mathrm{rad}]$ & $a\left[\frac{1}{\mathrm{~s}}\right]$ & $b\left[\frac{1}{\mathrm{~s}}\right]$ & $d\left[\frac{\mathrm{Nm}}{\mathrm{rad}}\right]$ & $e\left[\frac{\mathrm{Nms}}{\mathrm{rad}}\right]$ & $f[\mathrm{Nm}]$ \\
\hline 1.17 & -13.2 & 0.1 & -35.9 & -4.7 & 124.9 \\
\hline
\end{tabular}

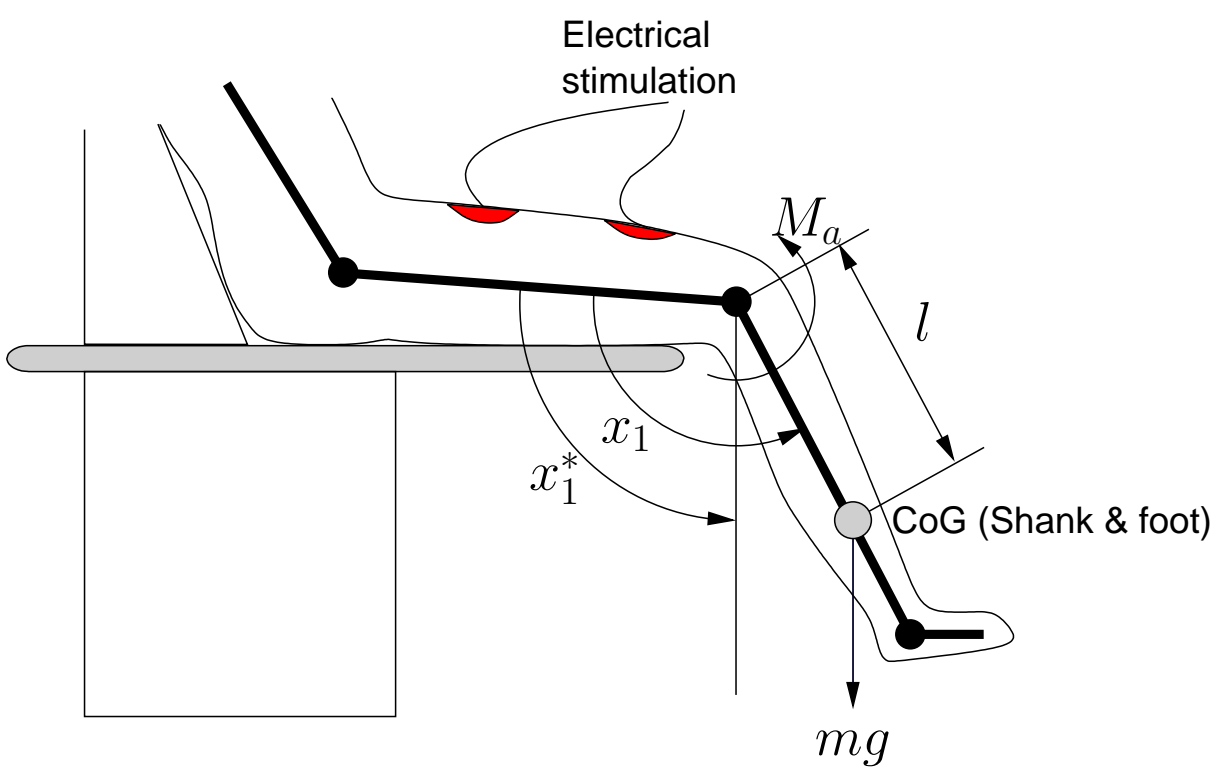

Fig. 1. Experimentel set-up. 


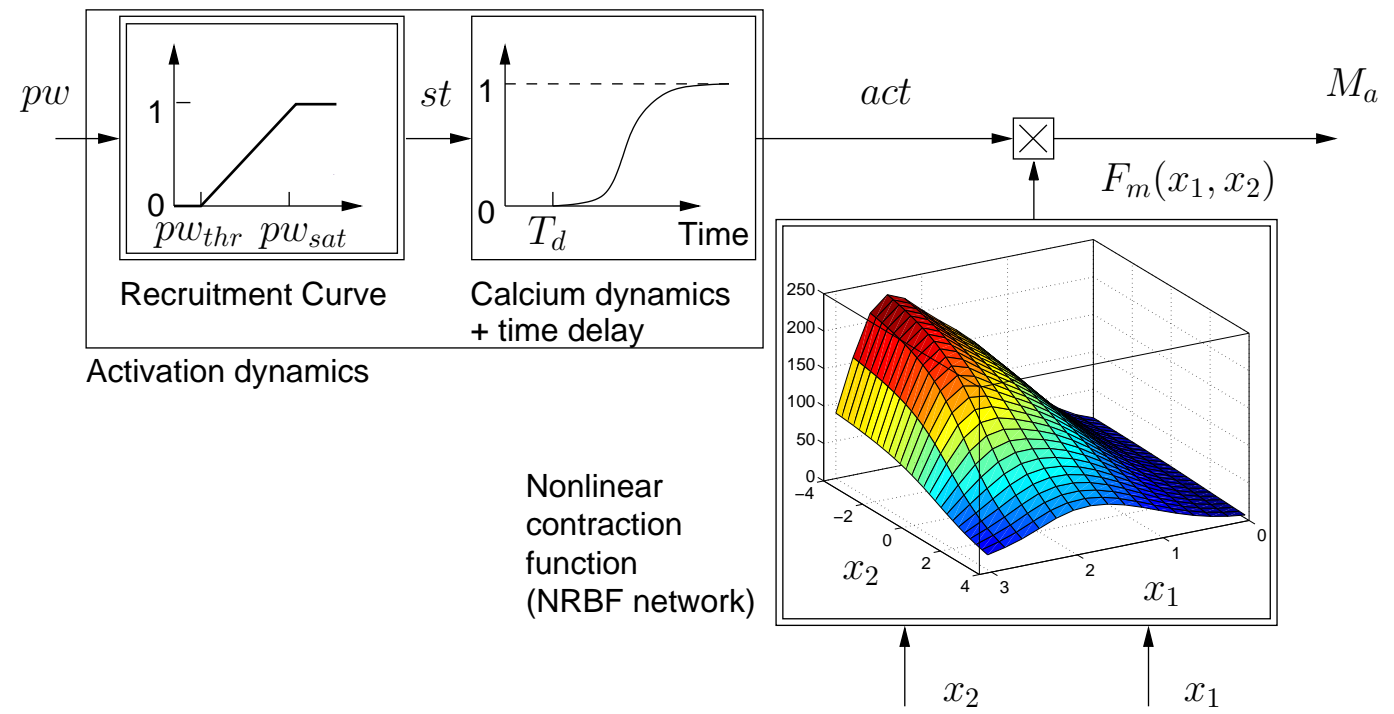

Fig. 2. Nonlinear model of the quadriceps muscle group.

Angle (plant simulator output) and its a priori EKF estimate (signals not distinguishable)
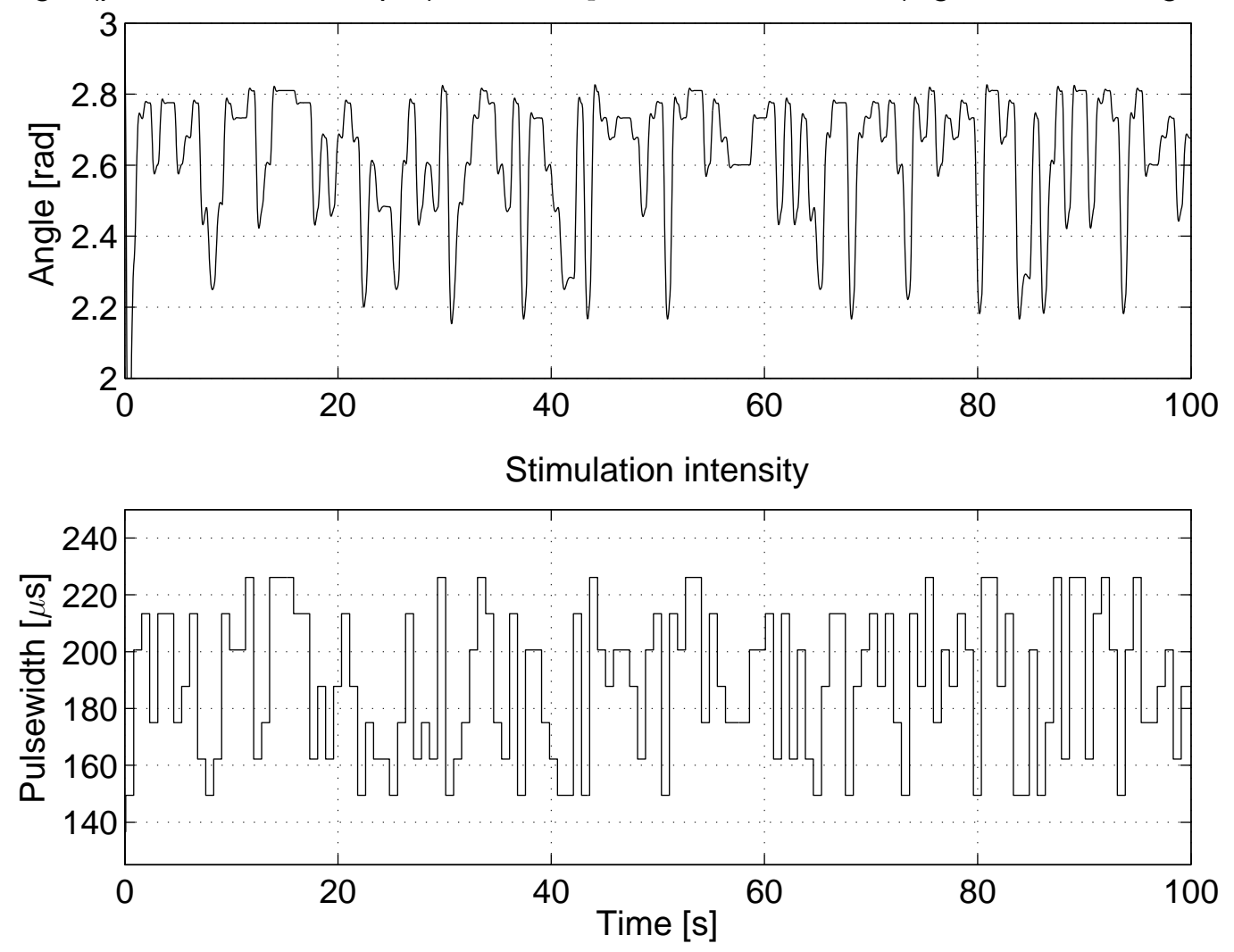

Fig. 3. Data set for online muscle identification. Data are generated by simulation using a complex physiologically-based model by Riener and Fuhr (1998). 
Active moment (dashed line) and its a priori EKF estimate (solid line)
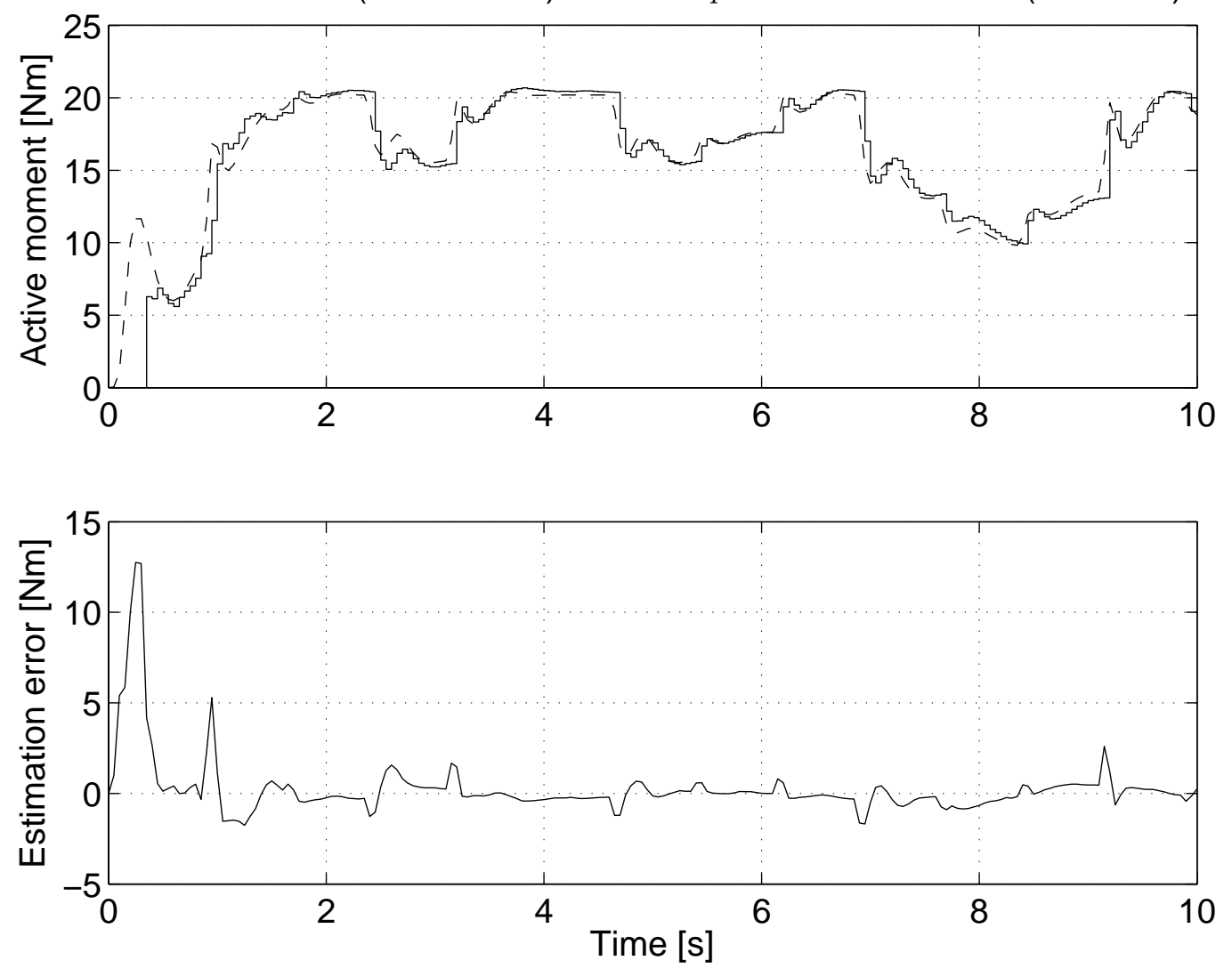

Fig. 4. One step ahead prediction of the active moment $\tilde{M}_{a}$ (a priori estimate of EKF) compared to the active moment $M_{a}$ inside the plant simulator. 

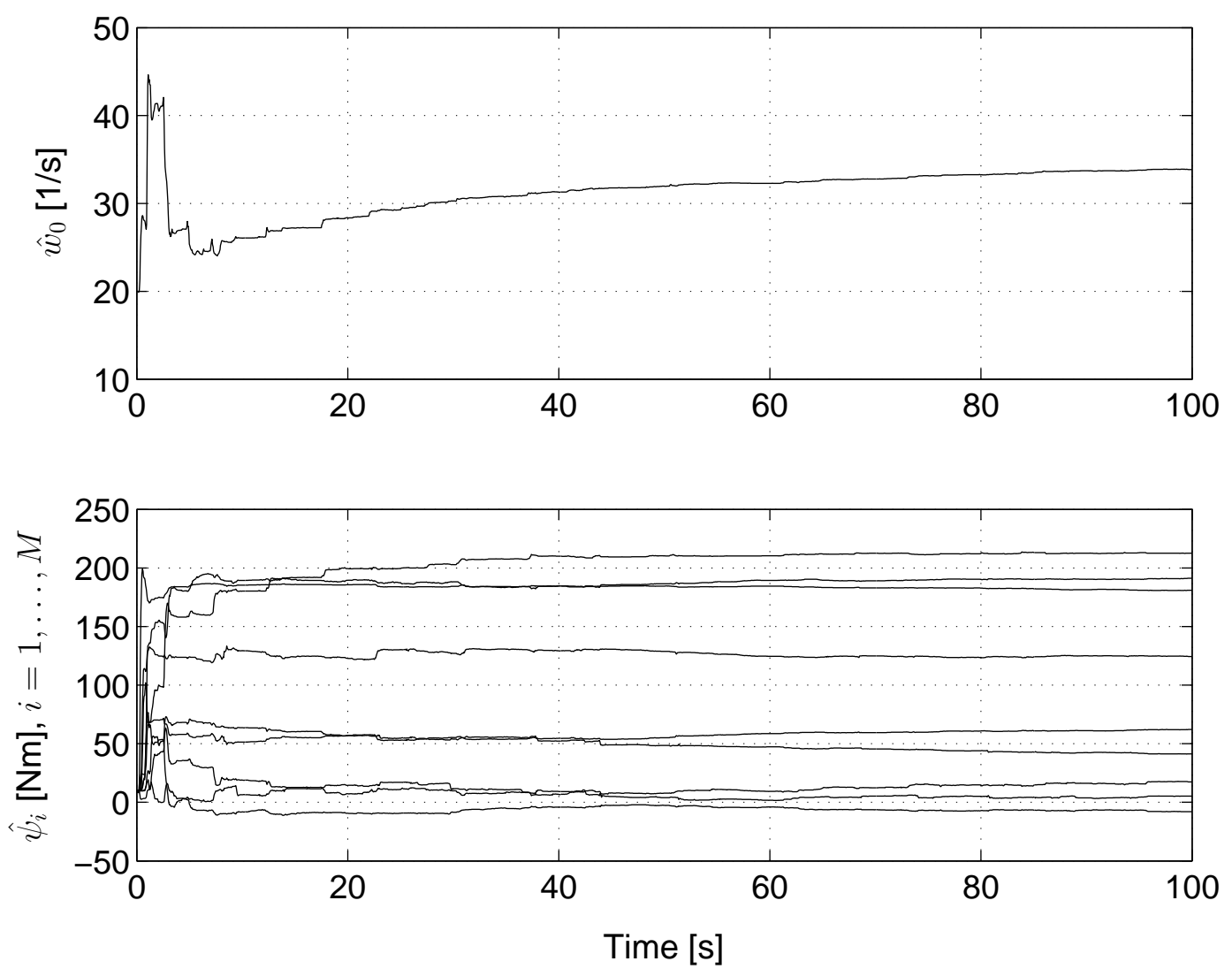

Fig. 5. Convergence of muscle parameter estimates for plant simulator identification. 


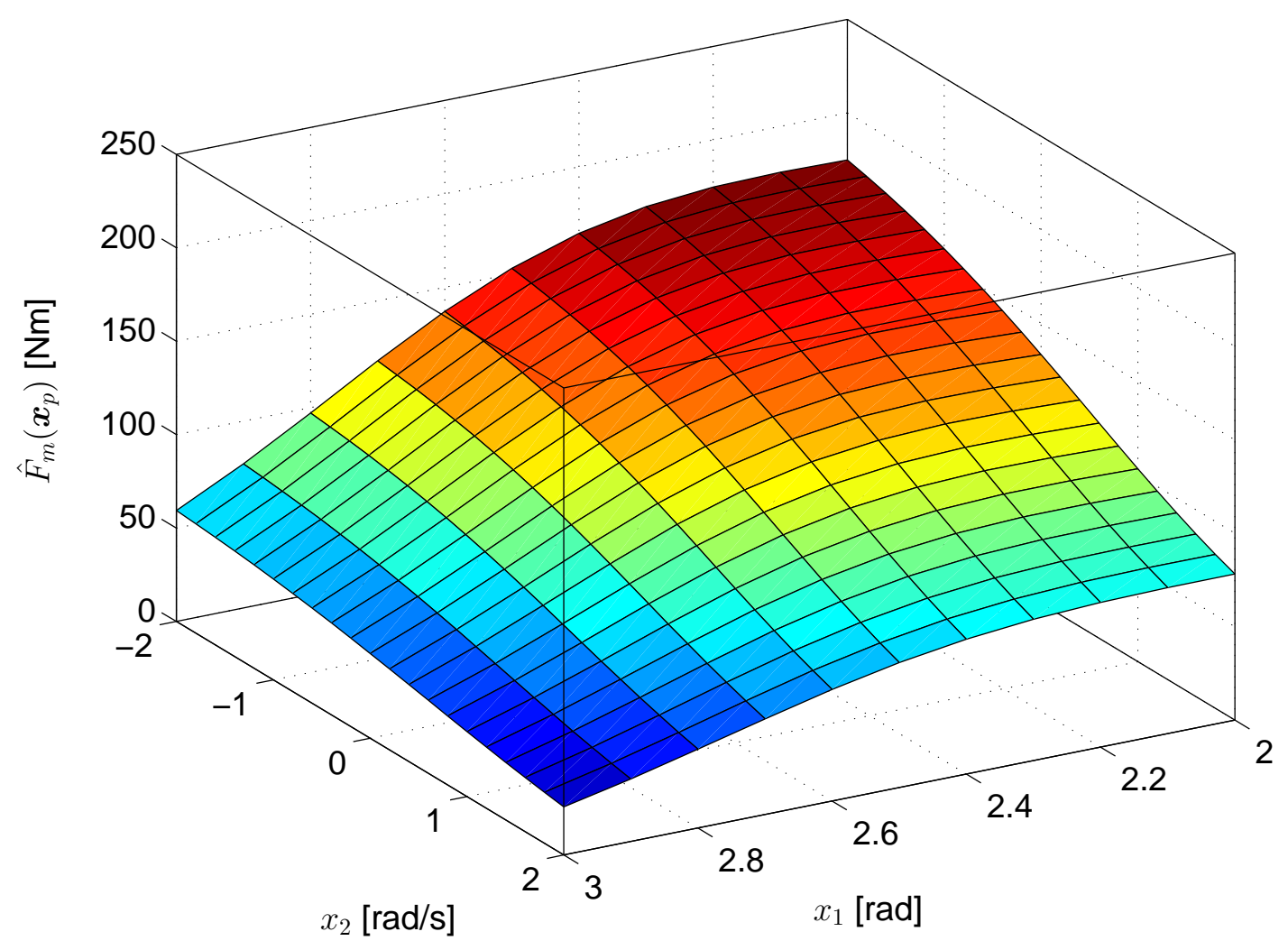

Fig. 6. Estimate of the nonlinear contraction function $\hat{F}_{m}\left(\boldsymbol{x}_{p}\right)$ for the plant simulator after $100 \mathrm{~s}$. 
Real angle (dashed line; plant simulator output) and simulated angle (bold line)
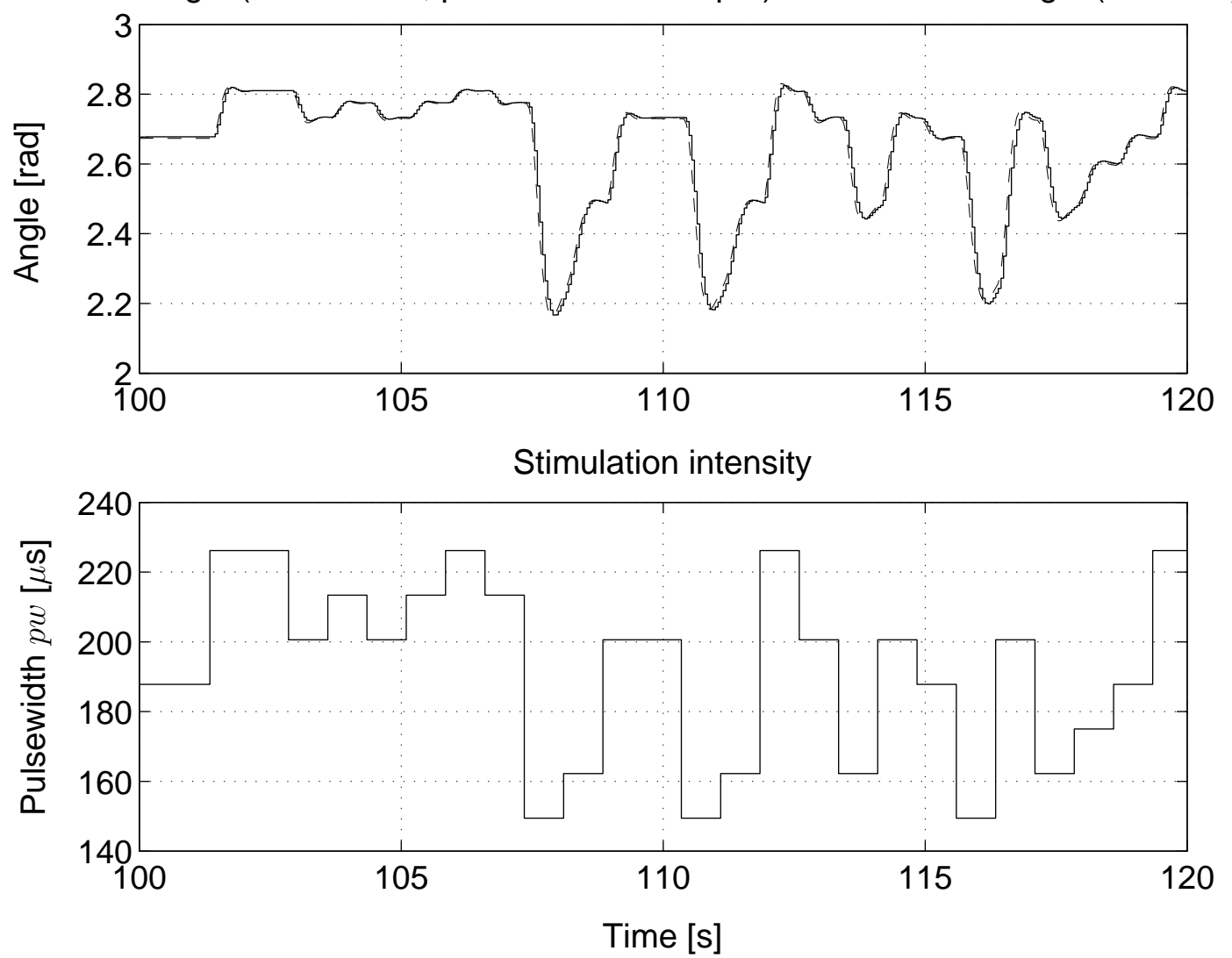

Fig. 7. Simulation test: The EKF is switched off and the estimated model runs only driven by the pulsewidth input. 
Real angle (bold line) and a priori angle estimate (thin line) (signals not distinguishable)
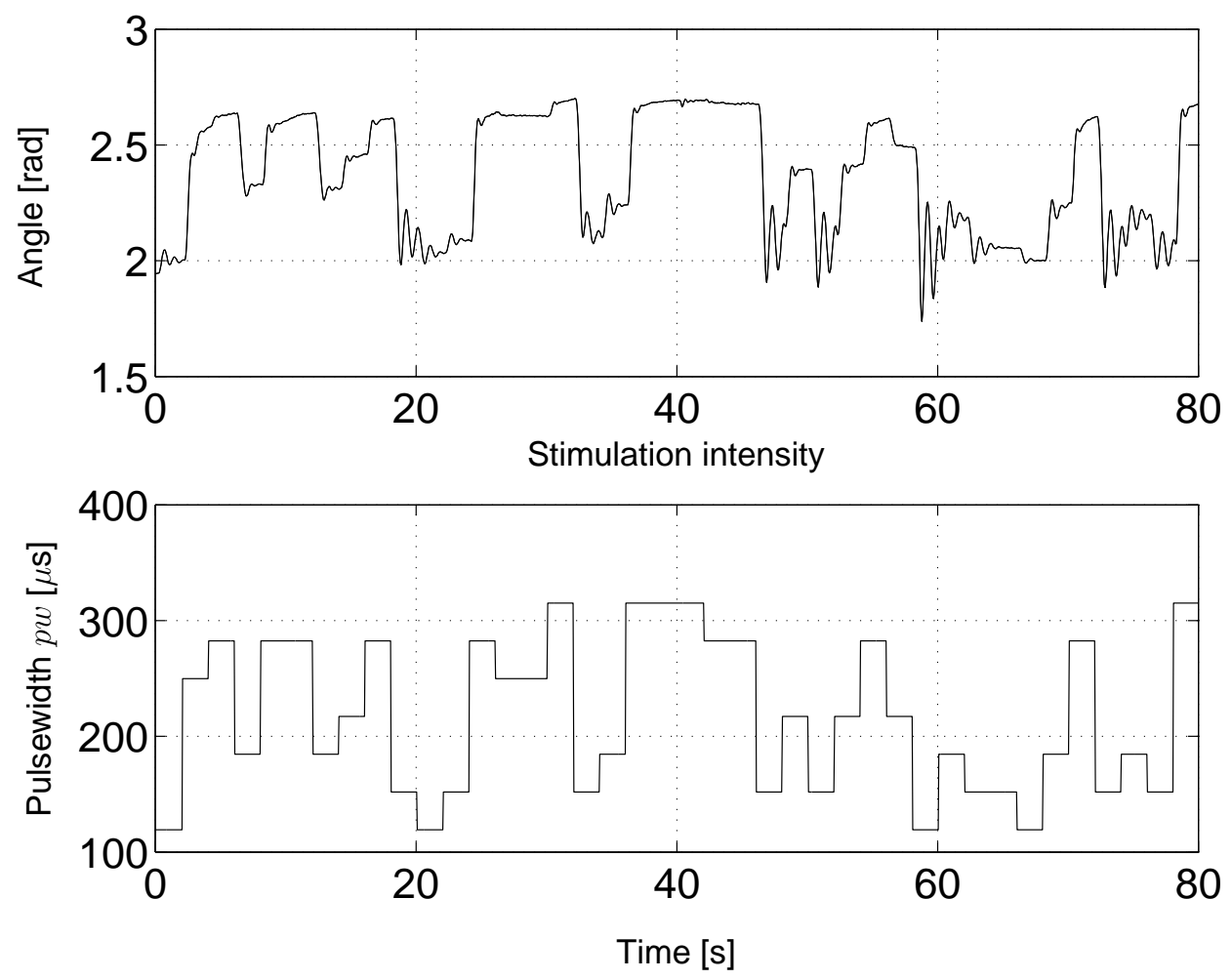

Fig. 8. I/O data set and a priori angle estimate for one paraplegic subject. 

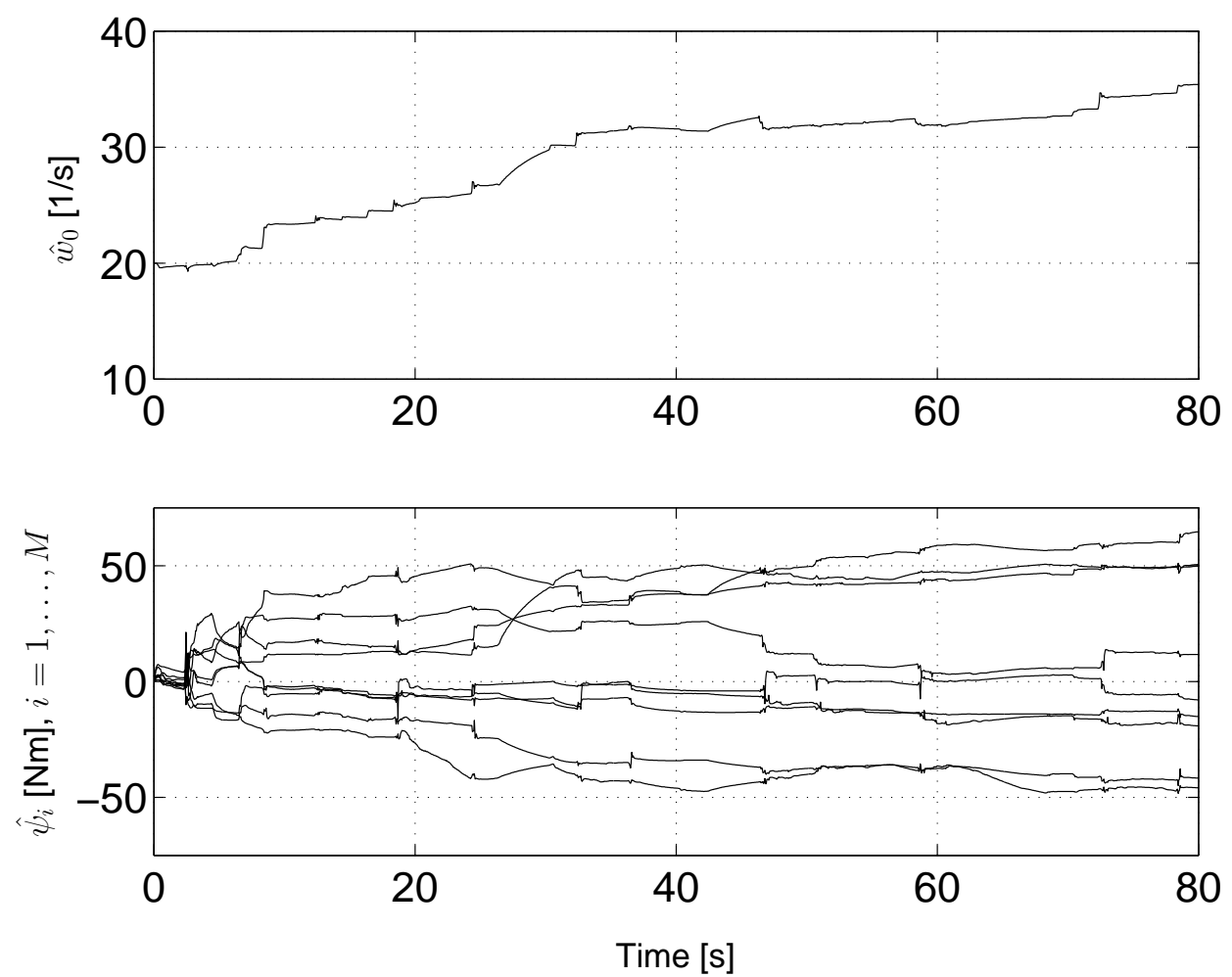

Fig. 9. Convergence of muscle parameters for running the EKF on experimental data. 
Real angle (bold line) and simulated angle (thin line)
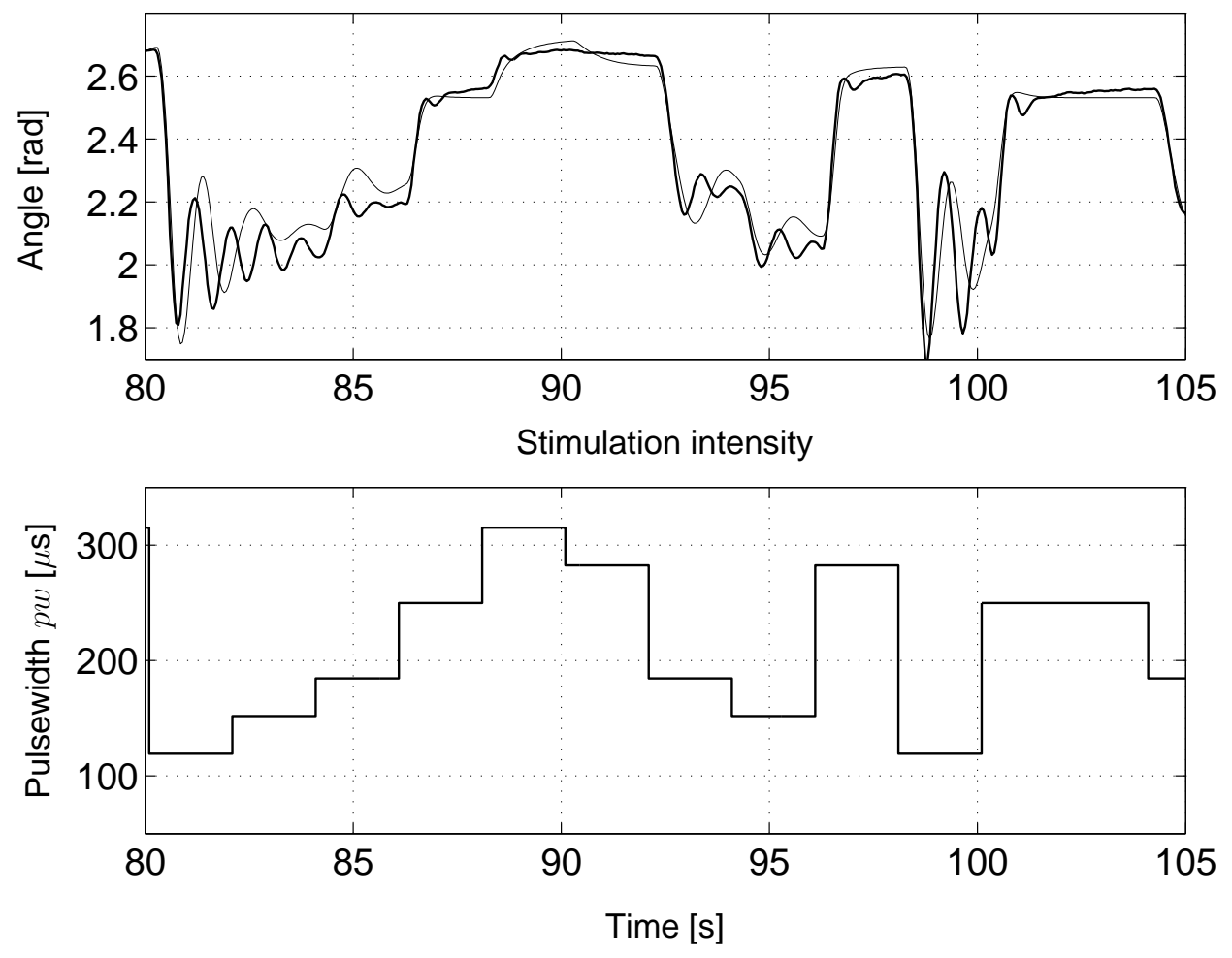

Fig. 10. Simulation test: The EKF is switched off and the estimated model runs only driven by the pulsewidth input. The simulated angle is plotted against the measured angle. 

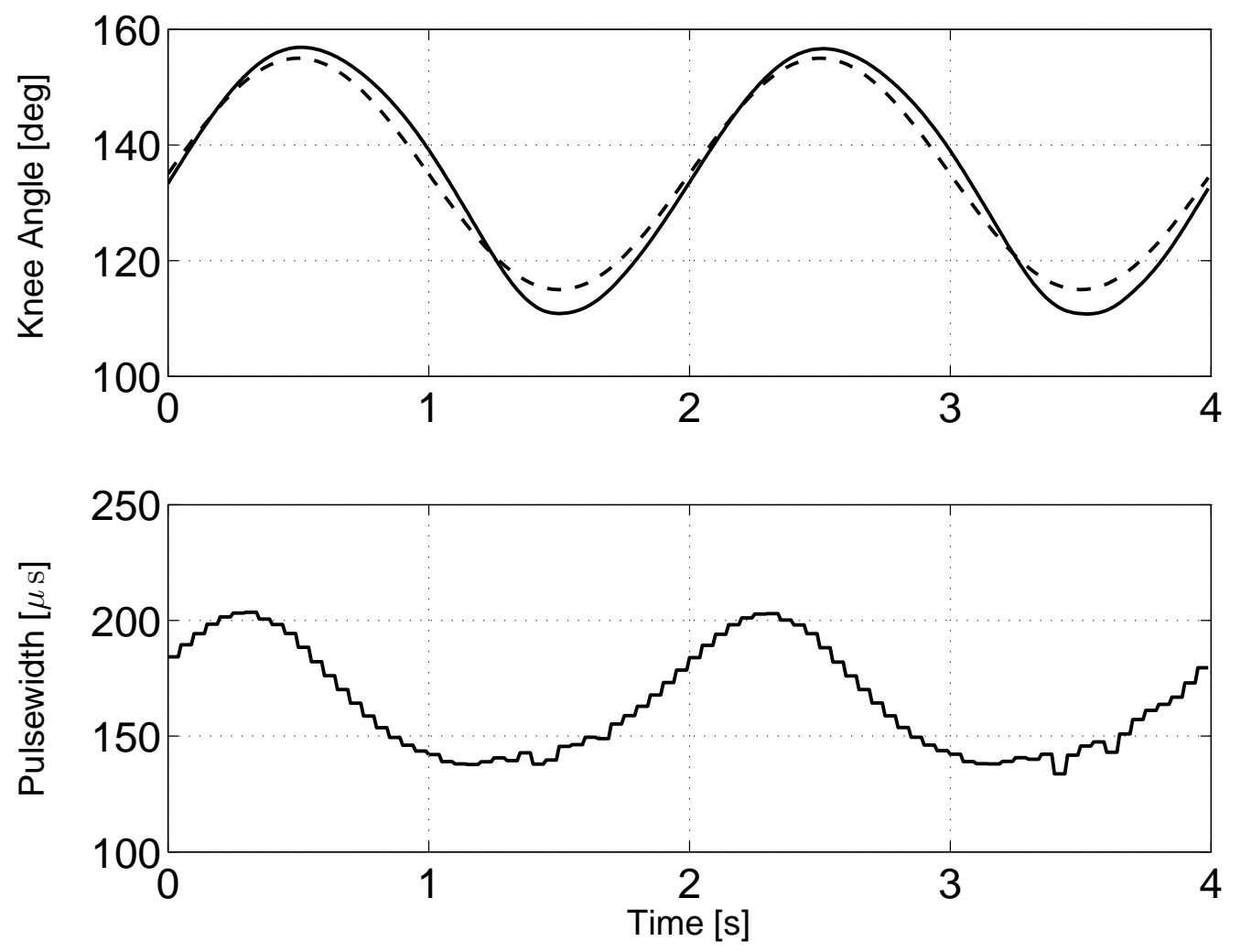

Fig. 11. Tracking test: The upper graph shows the reference angle (dashed line) and the controlled angle (solid line). The lower graph shows the control signal. 

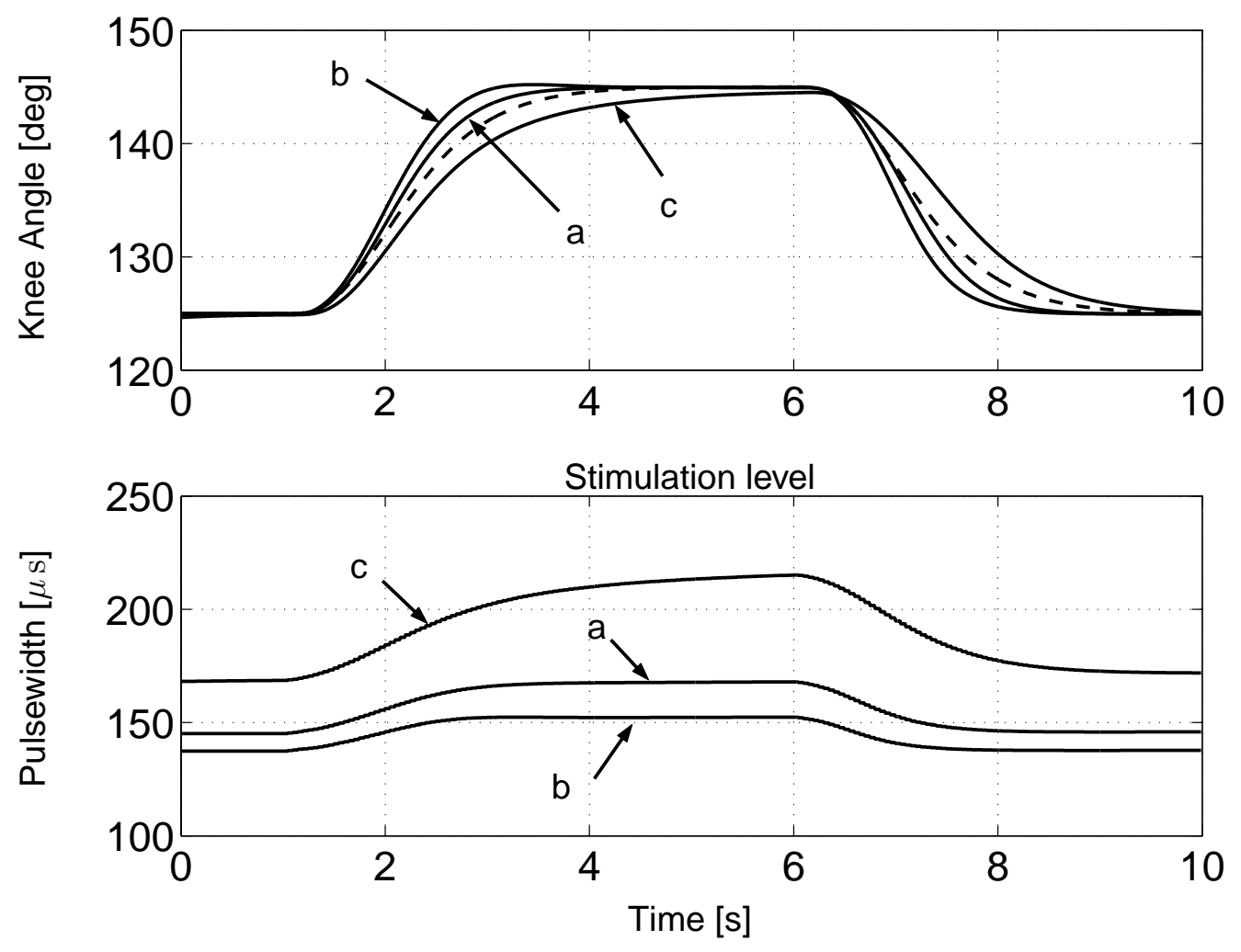

Fig. 12. Robustness tests: The upper graph shows the desired knee-joint angle (dashed line), and the closed-loop responses (solid lines) for the nominal and altered plant simulator; the following settings for the maximal isometric muscle force of the plant stimulator have been used: (a) 100\%, (b) $150 \%$ and (c) $50 \%$ of the nominal value. The lower graph shows the corresponding control signal. 